\title{
Crystal structure of L,D-transpeptidase $\mathrm{Ldt}_{\mathrm{Mt} 2}$ in complex with meropenem reveals the mechanism of carbapenem against Mycobacterium tuberculosis
}

Cell Research (2013) 23:728-731. doi:10.1038/cr.2013.53; published online 16 April 2013

\section{Dear Editor,}

Tuberculosis (TB), an infectious disease caused by the bacillus Mycobacterium tuberculosis, leads to substantial mortality worldwide [1]. The emergence of multidrugresistant (MDR) and extensively drug-resistant (XDR) strains of $M$. tuberculosis has challenged conventional anti-TB therapy and threatens global disease control of TB $[2,3]$. The development of new anti-TB drugs is urgently required [4]. $\beta$-lactams are effective antibiotics widely used to treat bacterial infections; however, so far no effective anti-TB antibiotics have emerged from this class of drugs. Previous studies have indicated that an important reason for the lack of effective $\beta$-lactam anti-TB antibiotics is the presence of a chromosomallyencoded class A (Ambler) $\beta$-lactamase, BlaC, in $M$. tuberculosis [5].

A recent study by Hugonnet et al. [6] has demonstrated that a combination of meropenem-clavulanate was effective against 13 XDR strains of $M$. tuberculosis. Meropenem is a member of the carbapenem class of $\beta$-lactams and contains a bicyclic nucleus, a pyrroline ring and a $\beta$-lactam ring, and clavulanate is a $\beta$-lactamase inhibitor. Both are FDA-approved drugs and could potentially be used to treat TB, providing a novel clinical treatment strategy [6]. However, the bactericidal mechanism of this drug combination in treating TB is unclear. Hugonnet et al. [6] suggest that one reason for the efficacy of the meropenem-clavulanate combination against $\mathrm{XDR}$ strains is that meropenem is poorly hydrolyzed by BlaC.

Carbapenems have been reported to target L,D-transpeptidases that generate the unusual $3 \rightarrow 3$ transpeptide linkages that are the predominant transpeptide linkages $(80 \%)$ in the peptidoglycan layer of nonreplicating $M$. tuberculosis $[7,8]$. L,D-transpeptidase type $2\left(\mathrm{Ldt}_{\mathrm{Mt2}}\right)$ is the main L,D-transpeptidase in M. tuberculosis and is critical for cell wall synthesis, virulence and amoxicillin tolerance of M. tuberculosis [9]. To kill M. tuberculosis effectively, the combination of meropenem-clavulanate not only inactivates $\beta$-lactamase but also prevents the formation of transpeptide linkages in the peptidoglycan layer [9]. As $\mathrm{Ldt}_{\mathrm{Mt}}$ is the enzyme that generates $80 \%$ of the transpeptide linkages in M. tuberculosis, the inactivation of $\mathrm{Ldt}_{\mathrm{Mt} 2}$ is likely to be the major mechanism underlying the effectiveness of meropenem-clavulanate against M. tuberculosis. Here, we report the crystal structures of an N-terminal-truncated $\mathrm{Ldt}_{\mathrm{Mt} 2}\left(\mathrm{Ldt}_{\mathrm{Mt} 2}-\Delta \mathrm{N} 54\right.$, residues 55-408), a trypsin-degraded fragment of $\mathrm{Ldt}_{\mathrm{Mt2}}\left(\mathrm{Ldt}_{\mathrm{Mt2}}{ }^{-}\right.$ $\Delta \mathrm{N} 139$, residues 140-408) and the complex of $\mathrm{Ldt}_{\mathrm{Mt}^{-}}$ $\Delta \mathrm{N} 139$ and meropenem, at 2.5, 1.8 and $1.4 \AA$ resolutions, respectively (Supplementary information, Figure $\mathrm{S} 1$ and Table S1). These structures reveal the structural mechanism through which meropenem may act to inhibit $\mathrm{Ldt}_{\mathrm{Mt2}}$.

Our apo $\mathrm{Ldt}_{\mathrm{Mt}}-\Delta \mathrm{N} 54$ structure (Figure $1 \mathrm{~A}$ ) shows the linear arrangement of the two N-terminal $\beta$-barrel domains (residues 60-148 and 149-250) and the C-terminal YkuD domain (residues 251-408). The two N-terminal $\beta$-barrel domains, both of which adopt an IgG-like fold, contain one three-stranded and one four-stranded sheet, respectively. We suggest that these two IgG-like domains act as a spacer arm for the YkuD catalytic domain. The YkuD domain is characterized by a $\beta$-sheet structure similar to that in $\mathrm{Ldt}_{\mathrm{Bs}}$ from Bacillus subtilis [10] and $\mathrm{Ldt}_{\mathrm{fm}}$ from Enterococcus faecium [11] (Supplementary information, Figure S1) with a root-mean-square deviation of $\mathrm{C} \alpha$ superposition of $1.6 \AA$ and $1.8 \AA$ and sequence identities of $20 \%$ and $24 \%$, respectively. As in $\operatorname{Ldt}_{\mathrm{Bs}}$ and $\mathrm{Ldt}_{\mathrm{fm}}$ $[10,11]$, the $\mathrm{YkuD}$ domain in $\mathrm{Ldt}_{\mathrm{M} t 2}$ contains a catalytic triad Cys354-His336-Ser337, and it is likely that residue Cys354 is directly involved in enzyme activity and is the target site for carbapenems. $\mathrm{Ldt}_{\mathrm{Mt} 2}$ also contains two additional segments, A (residues 300-323) and B (residues 379-408), which are not observed in $\mathrm{Ldt}_{\mathrm{Bs}}$ and $\mathrm{Ldt}_{\mathrm{fm}}$ (Supplementary information, Figure S1).

Co-crystallization of $\mathrm{Ldt}_{\mathrm{Mt} 2}-\Delta \mathrm{N} 54$ and meropenem failed, but co-crystallization of $\mathrm{Ldt}_{\mathrm{Mt2}}-\Delta \mathrm{N} 139$ and me- 

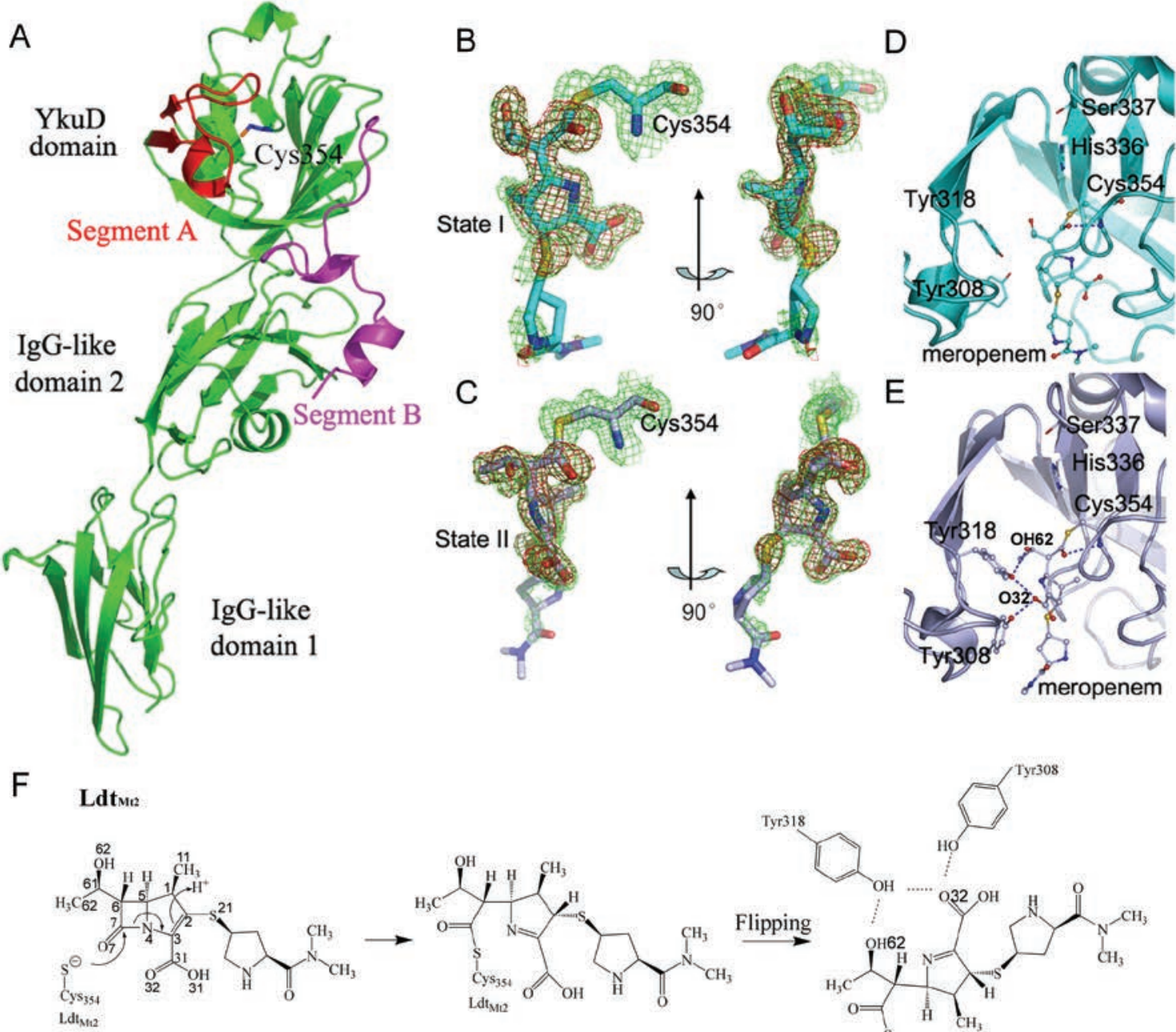

Enzyme and drug

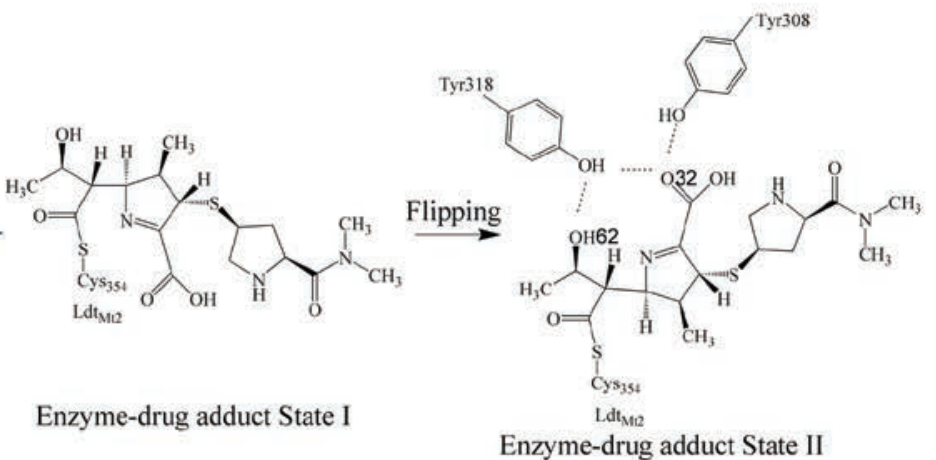

BlaC

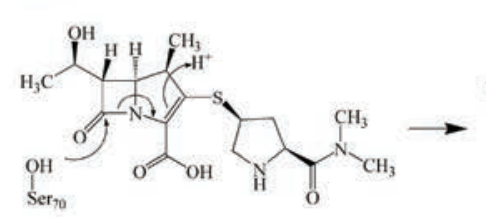

Enzyme and drug

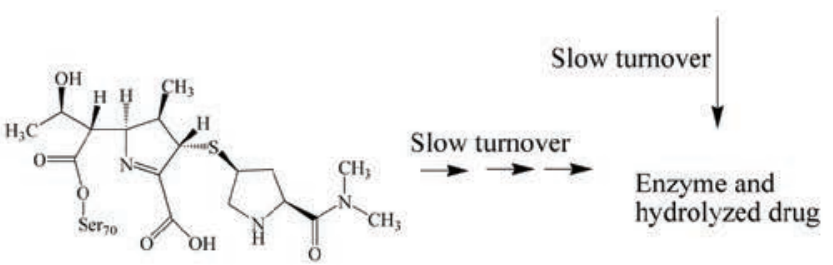

Enzyme-drug adduct

Figure 1 Structures of $\mathrm{Ldt}_{\mathrm{Mt} 2}$ and the $\mathrm{Ldt}_{\mathrm{Mt} 2}-$ meropenem complex. (A) Ribbon representation of the overall structure of $\mathrm{Ldt}_{\mathrm{Mt2}}{ }^{-}$ $\triangle \mathrm{N} 54$ (segments $\mathrm{A}$ and $\mathrm{B}$ are labeled in red and magenta, respectively). (B-E) Different conformations of meropenem in the $\mathrm{Ldt}_{\mathrm{Mt2}}-\Delta \mathrm{N} 139-$ meropenem complex. A 2Fo-Fc ligand-omitted electron density map (green, contour at $1 \sigma$ ), a Fo-Fc map (red, contour at $3 \sigma$ ), and ball-and-stick models of meropenems in State I (B, D, green) and II (C, E, gray) are shown. Hydrogen bonds are shown as blue dashed lines in $\mathbf{D}$ and $\mathbf{E}$. (F) Proposed mechanisms of the action of meropenem on Ldt $\mathrm{Mt}_{2}$ (the present study) and BlaC [4].

ropenem was successful. The asymmetric unit of $\mathrm{Ldt}_{\mathrm{Mt}^{2}}$ $\Delta \mathrm{N} 139$-meropenem co-crystals contains two $\mathrm{Ldt}_{\mathrm{Mt} 2^{-}}$ meropenem complexes. Meropenem adopts different conformations in the two complexes, hereafter referred to as State I (Figure 1B and 1D) and II (Figure 1C and $1 \mathrm{E})$. In both states, meropenem is covalently linked to 
the catalytic residue Cys354 via thioester formation. The carbapenem nucleus of meropenem appears clearly, displays well-defined electron densities and shows the same conformation in both State I and II (Figure 1B and 1D). The planar arrangement of atoms $\mathrm{C} 2, \mathrm{C} 3, \mathrm{~N} 4, \mathrm{C} 5$, and $\mathrm{C} 31$ of meropenem in complex with $\mathrm{Ldt}_{\mathrm{Mt} 2}$ results in the appearance of a double bond between $\mathrm{C} 3$ and $\mathrm{N} 4$ (Figure $1 \mathrm{~F}$ ), replacing the $\mathrm{C} 2 / \mathrm{C} 3$ double bond observed in meropenem $[12,13]$. The $\mathrm{C} 3 / \mathrm{N} 4$ double bond was also observed in meropenem covalently linked to $\mathrm{BlaC}$ [6] and other $\beta$-lactamases [13]. This tautomerism of the carbapenem nucleus is accompanied by the covalent linking of meropenem to $\mathrm{Ldt}_{\mathrm{M} 12}$. As tautomerization of the carbapenem nucleus has been reported to cause slow turnover in carbapenem hydrolyzation by $\mathrm{BlaC}[6]$ and other $\beta$-lactamases [13], we propose that tautomerization helps to stablize the $\mathrm{Ldt}_{\mathrm{Mt} 2}$-meropenem adduct.

Despite these similarities, the two states are distinct from each other. In State I, atom C7 of meropenem (Figure $1 \mathrm{~F}$ and Supplementary information, Figure S2) is close to atom N4 at a distance of $2.87 \AA$. This conformation is very similar to that reported previously for meropenem in complex with BlaC [6], and likely represents the initial step during the action of meropenem on $\mathrm{Ldt}_{\mathrm{Mt} 2}$. In State II, the carbapenem nucleus has flipped approximately 180 degrees around the single bond connecting atoms C5 and C6 (Supplementary information, Figure S3), resulting in a larger C7-N4 distance of 3.21 $\AA$.

Interestingly, by flipping the carbapenem nucleus in State II, residue Tyr308 of $\mathrm{Ldt}_{\mathrm{Mt} 2}$ adopts a different rotamer conformation by rotating approximately 90 degrees around the $\mathrm{C} \alpha / \mathrm{C} \beta$ bond (Supplementary information, Figure S3). We suggest that rotating of the Tyr308 side chain in $\mathrm{Ldt}_{\mathrm{Mt} 2}$ and flipping of the carbapenem nucleus in meropenem are the second step following the $\beta$-lactam ring opening in the first step during the action of meropenem on $\mathrm{Ldt}_{\mathrm{M} t 2}$, and result in three additional hydrogen bonds $\left({ }^{\mathrm{Tyr} 318} \mathrm{OH}-{ }^{\mathrm{Mer}} \mathrm{OH} 62,{ }^{\mathrm{Ty} 3318} \mathrm{OH}-{ }^{\mathrm{Mer}} \mathrm{O} 32\right.$, and ${ }^{\mathrm{Ty} r 308} \mathrm{OH}-$ ${ }^{\mathrm{Mer}} \mathrm{O} 32$ ) between the enzyme and meropenem in State II (Figure 1E and Supplementary information, Figure S3). In contrast, only one hydrogen bond $\left({ }^{\mathrm{Gly} 353} \mathrm{~N}-{ }^{\mathrm{Mer}} \mathrm{O} 7\right)$ is observed in State I (Figure 1D). Residues Tyr308 and Tyr318 that form hydrogen bonds with meropenem are conserved in $\mathrm{Ldt}_{\mathrm{M}+2}$ and its homologs, but not in other L,D-transpeptidases (Supplementary information, Figure S1). Mutant protein Y318F or Y318A hydrolyzes meropenem at a faster rate than the wild-type protein (Supplementary information, Figure S4), supporting that the additional hydrogen bonds are important for stabilization of the enzyme-drug adduct and indicating that the flipping of meropenem and the resulting additional hydrogen bonds are involved in the mechanism underlying the inhibition of $\mathrm{Ldt}_{\mathrm{Mt} 2}$ by meropenem (Figure $1 \mathrm{~F}$ ).

As a main peptidoglycan-crosslinking L,D-transpeptidase, $\mathrm{Ldt}_{\mathrm{M} t 2}$ is an important target in the development of drugs against XDR M. tuberculosis [9, 14]. Our analyses of the $\mathrm{Ldt}_{\mathrm{Mt}}$-meropenem complex structure reveal a twostep mechanism of drug actions (Figure $1 \mathrm{~F}$ top). The first step involves opening the $\beta$-lactam ring of meropenem and formation of a thioester between meropenem and $\mathrm{Ldt}_{\mathrm{M} \mathrm{t} 2}$, accompanied by the simultaneous tautomerization of the $\mathrm{C} 2 / \mathrm{C} 3$ double bond to $\mathrm{C} 3 / \mathrm{N} 4$ double bond (State I). These events of the first step are also observed in the complex of meropenem and BlaC [6] (Figure 1F bottom). During the second step, the C5/C6 single bond of meropenem rotates approximately 180 degrees, and this rotation induces localized structural changes in segment A (including residues Tyr308 and Tyr318) of $\mathrm{Ldt}_{\mathrm{Mt}}$, leading to the formation of several hydrogen bonds (State II), stabilizing the structure of the $\mathrm{Ldt}_{\mathrm{Mt} 2}$-meropenem adduct, and resulting in a slow turnover in drug hydrolysis. This mechanism may be shared by carbapenems, such as meropenem and imipenem, which have been reported to inhibit $\mathrm{Ldt}_{\mathrm{Mt} 2}$.

Furthermore, we explored the role of tautomerization in stabilizing the complex of $\mathrm{Ldt}_{\mathrm{Mt} 2}$ and antibiotics. When we performed mass spectrometry assays with cephems such as cepholotin and cefuroxime, a subclass of $\beta$-lactams that do not inhibit $\mathrm{Ldt}_{\mathrm{Mt} 2}$, we found that cephems also form covalent adducts with $\mathrm{Ldt}_{\mathrm{M} 12}$ (Supplementary information, Table S2 and Figure S6). We thus propose that the mechanism of $\mathrm{Ldt}_{\mathrm{Mt} 2}$ action on cephems is the same as that of $\mathrm{Ldt}_{\mathrm{Mt} 1}$ action on cephems [14] and involves a tautomerization of the cephem nucleus (a $\beta$-lactam ring and a dihydrothiazine ring) (Supplementary information, Figure S5A). As cephems cannot inhibit $\mathrm{Ldt}_{\mathrm{Mt}}$, we suggest that tautomerization alone is not sufficient to stabilize the adduct of $\mathrm{Ldt}_{\mathrm{Mt} 2}$ and cephems. To stabilize the adduct of $\mathrm{Ldt}_{\mathrm{Mt} 2}$ and carbapenem (or any other potential inhibitor of $\mathrm{Ldt}_{\mathrm{M} t 2}$ ), tautomerization is necessary but not sufficient, and the conformational change in the second step likely also plays an important role. The results reported here, together with the reported structure of the BlaC-meropenem complex [15], reveal the structural basis for the stabilization of enzymecarbapenem adducts and may suggest new strategies for the design of antibiotics derived from $\beta$-lactams to fight against XDR M. tuberculosis.

\section{Accession codes}

Coordinates and structure factor files of $\mathrm{Ldt}_{\mathrm{Mt2}}-\Delta \mathrm{N} 54, \mathrm{Ldt}_{\mathrm{Mt2}}{ }^{-}$ $\Delta \mathrm{N} 139$, and the $\mathrm{Ldt}_{\mathrm{Mt} 12}-\Delta \mathrm{N} 139$-meropenem complex have been deposited in the Protein Data Bank with accession codes 3 VYN, 3 VYO and 3 VYP. 


\section{Acknowledgments}

We are grateful for the assistance of $\mathrm{P} \mathrm{Wu}$ in mass spectrometry analysis and M Fan in crystallographic data collection. We thank the staff at beamline 17A, KEK, Photon Factory, Japan, and beamline 17U, Shanghai Synchrotron Radiation Facility, for assistance with data collection. This work was funded by National Basic Research Program of China (973 Program, 2011CB910300, 2011CB911103 and 2013CB911500) and the Chinese Academy of Sciences (KSCX2-EW-J-3)

\section{Wen-Juan $\mathrm{Li}^{1,2,{ }^{1}}$, De-Feng $\mathrm{Li}^{1, *}$, Yong-Lin $\mathrm{Hu}^{1}$, Xian-En Zhang ${ }^{1}$, Li-Jun Bi ${ }^{1}$, Da-Cheng Wang ${ }^{1}$}

${ }^{I}$ National Laboratory of Biomacromolecules and Laboratory of Noncoding RNA, Institute of Biophysics, Chinese Academy of Sciences, Beijing 100101, China; ${ }^{2}$ University of the Chinese Academy of Sciences, Beijing 100049, China

*These two authors contributed equally to this work.

Correspondence: Li-Jun $\mathrm{Bi}^{\mathrm{a}}$, Da-Cheng Wang ${ }^{\mathrm{b}}$

${ }^{\mathrm{a}}$ Tel: +86-10-64888464; Fax: +86-10-64871293

E-mail: blj@ibp.ac.cn

${ }^{\mathrm{b}}$ Tel: +86-10-64888547; Fax: +86-10-64888560

E-mail: dcwang@ibp.ac.cn

\section{References}

1 Dye C, BG Williams. Science 2010; 328:856-861.

2 WHO global tuberculosis control report 2010. Summary. Cent Eur J Public Health 2010; 18:237.

3 Gandhi NR, Nunn P, Dheda K, et al. Lancet 2010; 375:1830-1843.

4 Koul A, Arnoult E, Lounis N, et al. Nature 2011; 469:483-490.

5 Cole ST, Brosch R, Parkhill J, et al. Nature 1998; 393:537-544.

6 Hugonnet JE, Tremblay LW, Boshoff HI, et al. Science 2009; 323:1215-1218.

7 Lavollay M, Arthur M, Fourgeaud M, et al. J Bacteriol 2008; 190:4360-4366.

8 Wietzerbin J, Das BC, Petit JF, et al. Biochemistry 1974; 13:34713476.

9 Gupta R, Lavollay M, Mainardi JL, et al. Nat Med 2010; 16:466-469.

10 Bielnicki J, Devedjiev Y, Derewenda U, et al. Proteins 2006; 62:144151.

11 Biarrotte-Sorin S, Hugonnet JE, Delfosse V, et al. J Mol Biol 2006; 359:533-538.

12 Drawz SM, RA Bonomo. Clin Microbiol Rev 2010; 23:160-201.

13 Therrien C, RC Levesque. FEMS Microbiol Rev 2000; 24:251-262.

14 Dubée V, Triboulet S, Mainardi JL, et al. Antimicrob Agents Chemother 2012; 56:4189-4195.

15 Tremblay LW, JE Hugonnet, JS Blanchard. Biochemistry 2008; 47:5312-5316.

(Supplementary information is linked to the online version of the paper on the Cell Research website.) 